\title{
Nicotine replacement: a new approach to reducing tobacco-related harm
}

\author{
C. Jiménez-Ruiz*, M. Kunze**, K.O. Fagerström+
}

Nicotine replacement: a new approach to reducing tobacco-related harm. C. Jiménez-Ruiz, M. Kunze, K.O. Fagerström. OERS Journals Ltd 1998.

ABSTRACT: Primary prevention is usually regarded as the most desirable goal in efforts to control tobacco-related diseases. However, this has not been very effective so far; moreover, it would take 30-40 yrs for primary prevention to translate into major health benefits. Modification of tobacco products and/or reduction of tobacco use may also have some impact on tobacco-related diseases. A tobacco dose-dependent risk has been observed in these diseases, including cancer, cardiovascular diseases, chronic nonspecific respiratory disorders, and problems during pregnancy.

Reduced smoking (smoking fewer cigarettes, leading to a reduced intake of toxic substances) may be indicated in individuals who: 1 ) are failing in cessation attempts; 2) want to quit but are unable to do so; and 3) do not want to quit but want to reduce smoking. Studies have shown that nicotine replacement medications may be an untapped source in efforts to reduce smoking.

Based on Austrian data, it is estimated that, approximately 10 yrs after implementation, a 1\% reduction in smoking could save 14 male lung cancer deaths each year, and a $50 \%$ reduction would save 700 male lives. Inclusion of females and other tobacco-related diseases suggest that thousands of lives could be saved if smoking could be reduced by $50 \%$. In the European Union, such a reduction in smoking could save $\mathbf{S 1 0 0 , 0 0 0}$ lives annually. Even a $1 \%$ reduction would save 1,000 lives.

In conclusion, reduced smoking should be explored as a valid method of reducing tobacco-related harm in those unwilling or unable to quit smoking.

Eur Respir J 1998; 11: 473-479.
*Smoking Unit, Dept of Pneumology, Hospital de la Princesa, Madrid, Spain. **Institute of Social Medicine, University of Vienna, Vienna, Austria. ${ }^{+}$Dept of Pulmonary Medicine, Helsingborg Hospital and Pharmacia \& Upjohn Consumer Healthcare, Helsingborg, Sweden.

Correspondence: C. Jiménez-Ruiz

Smoking Unit

Dept of Pneumology

Hospital de la Princesa

C/Diego de Leon 62

E-280 06 Madrid

Spain

Fax: 3414013582

Keywords: Nicotine nicotine replacement smoking

Received: March 261997

Accepted after revision July 311997
Each year, approximately 3 million smokers die prematurely because of cigarette smoking [1], and health professionals must take into account that 30-70\% of these deaths are avoidable [1]. There are three main ways of dealing with this problem.

Primary prevention. By definition this appears to be the optimal solution, since it would eradicate the problem in approximately 50 yrs. However, there are two drawbacks: no primary prevention programme has so far been effective [2]; and it would take 30-40 yrs for any major health benefits to occur, because young people smoking today will develop tobacco-related disease in future years.

Modification of tobacco products. Theoretically, this offers an opportunity to reduce tobacco-related harm. Switching from high- to low-tar cigarettes appears to reduce harm to some degree [3], and new types of cigarette, such as Premier® and Eclipse ${ }^{\circledR}[4]$, that heat, rather than burn, tobacco, could be beneficial as long as their use does not deter smokers from quitting. The most effective forms of modification are those products that do not heat or burn tobacco, i.e. smokeless tobacco. Some such preparations, e.g. moist snuff ("snus") used in Sweden, do not appear to cause oral or other cancers [5]. In addition, snuff appears

For editorial comments see page 263 much less likely to cause cardiovascular disease than cigarette smoking $[6,7]$.

Smoking reduction and cessation. Many smokers want to quit, and a recent European study conducted in Denmark, France, Germany, Spain and the UK reported that 5 and $14 \%$ of smokers intend to quit in the next 1 and 6 months, respectively [8]. However, only $2-3 \%$ of smokers attempting to quit finally succeed [9], and only about half of those who have ever smoked can quit during their lifetime, primarily because of nicotine dependence. Thus, the majority of motivated quitters fail [10]. This inability to control tobacco use may become distressing if the smoker develops a tobacco-related disease, such as chronic obstructive pulmonary disease (COPD).

Although many smokers want to quit, it appears that more smokers want to reduce the number of cigarettes smoked daily. Indeed, in the European study above, 39\% of respondents stated that they were trying to reduce smoking [8]. Although physicians have often advised patients to smoke less, this advice has seldom had any lasting effect. This is probably a result of the nicotine dependence seen in most smokers, which causes them to return to their preferred blood nicotine levels after a period of suffering mild withdrawal symptoms. If most smokers need to obtain nicotine, but not the tar, carbon monoxide (CO) and other substances contained in cigarette smoke [11], then 
this presents an opportunity to reduce tobacco-related harm by supplying nicotine in the form of nicotine medications [12]. Thus, encouraging the large proportion of smokers who want to reduce smoking to do so could complement cessation programmes by improving public health and the status of patients with tobacco-related disease, e.g. COPD [13]. For patients with COPD who cannot or do not want to quit, it is a medical imperative to help them to reduce exposure to the toxic substances in cigarette smoke.

\section{Does reduced smoking result in less harm?}

\section{Cancer}

Cigarette smoking is the principal cause of cancer of various organs, including the lung, larynx, oral cavity and oesophagus. Smoking has also been shown to contribute to cancer of the bladder, kidney, pancreas, liver, stomach, anus, penis, vulva and cervix [14, 15]. For cancers of the lung, larynx, oral cavity and oesophagus, there is a dosedependent relationship between cigarette consumption and risk [16]; those who smoke Š40 cigarettes $\cdot$ day $^{-1}$ have double the risk of developing lung cancer compared to those who smoke ð20 cigarettes day $^{-1}$ [17]. Similarly, those who smoke ð10 cigarettes.day-1 have a fourfold reduction in risk [17] (table 1).

A dose-dependent relationship has also been noted in cancer of the pancreas, stomach, liver, cervix, anus, vulva and penis [16, 18-23]. A recent study of patients with grade 1 cervical intra-epithelial neoplasia reported a significant correlation between the extent of smoking reduction and the change in lesion size [24].

Overall, smoking $>20$ cigarettes $\cdot$ day $^{-1}$ corresponds with an increased risk of developing cancer, with those who smoke Š40 cigarettes $\cdot$ day $^{-1}$ at greatest risk. It appears that when heavy smokers quit, they experience a smaller reduction in the incidence of lung cancer than light or moderate smokers [16]. One study of the possible cancer marker anti-benzo(a)pyrene diolepoxide-deoxyribonucleic acid (DNA) found that, in addition to cessation, a 50\% reduction in smoking also altered the antibody response to this marker [25].

\section{Pulmonary disease}

Cigarette smoking is also the principal cause of chronic nonspecific respiratory symptoms (e.g. cough, phlegm production, dyspnoea, wheezing, etc.) and of COPD [24]. The number of cigarettes smoked daily correlates strongly with the risk of developing respiratory diseases, with heavy

Table 1. - Risk of developing lung cancer as a function of the number of cigarettes smoked daily [17]

\begin{tabular}{lc}
\hline${\text { Cigarettes } \cdot \text { day }^{-1} \mathrm{n}}$ & Relative risk \\
\hline 0 (nonsmoker) & 1.0 \\
$1-10$ & 5.5 \\
$11-19$ & 11.2 \\
20 & 14.2 \\
$21-31$ & 20.4 \\
$>31$ & 22.0 \\
\hline
\end{tabular}

Table 2. - Annual decrease in forced expiratory volume in one second $\left(\triangle F E V_{1}\right)$ as a function of the number of cigarettes smoked daily [28]

\begin{tabular}{lc}
\hline${\text { Cigarettes } \cdot \text { day }^{-1} \mathrm{n}}^{-}$ & $\Delta \mathrm{FEV}_{1} \mathrm{~mL}$ \\
\hline 0 & 36 \\
$1-4$ & 44 \\
$5-15$ & 46 \\
$16-20$ & 54 \\
\hline
\end{tabular}

smokers more likely to experience chronic nonspecific respiratory symptoms than light or moderate smokers [26]. A dose-dependent relationship between COPD and cigarette consumption has been shown [27]. In longitudinal studies, the annual loss of forced expiratory volume in one second (FEV1) among smokers depended on the number of cigarettes smoked daily [28] (table 2). These results have been confirmed by several population-based longitudinal studies in different countries [28]. DOCKERY et al. [29] analysed spirometric measurement in 8,191 smokers from six cities in the USA and found that for each pack-year smoked the mean loss of FEV1 was $7.4 \mathrm{~mL}$ for males and $4.4 \mathrm{~mL}$ for females [29]. Heavier cigarette consumption correlates with a higher prevalence of small airways obstruction and a lower capacity for diffusion [26]. Thus, although consumption of even a few cigarettes per day poses a risk for the pulmonary airways, smoking S20 cigarettes $\cdot$ day $^{-1}$ causes most harm [26-28].

\section{Cardiovascular disease}

Hypertension, hypercholesterolaemia and smoking constitute the main risk factors for cardiovascular disease [30]. Smokers have a greater risk of suffering from coronary heart disease and cerebrovascular disease than nonsmokers. Studies of cigarette smoking and cardiovascular disease have also indicated a dose-dependent risk relationship; the degree of risk is related to the number of cigarettes smoked (cumulative consumption), with heavier smoking associated with higher risks of myocardial in-farction, re-infarction and death $[18,30]$.

Smoking has also been identified as an important risk factor for peripheral vascular disease and abdominal aortic aneurysm [30]. In addition, smoking $>15$ cigarettes $\cdot$ day-1 reduces limb salvage rates; patients who smoke $>15$ cigarettes $\cdot$ day $^{-1}$ have much higher amputation rates following arterial reconstruction than those who smoke $<15$ cigarettes. day ${ }^{-1}$ [30]. Winiwarter-Buerger disease (thromboangiitis obliterans), an inflammatory and obliterative disease of the blood vessels of the extremities leading to tissue ischaemia and gangrene, is caused by cigarette smoking, and can only be treated by quitting [30]. However, no clear dose-dependent risk has been shown between smoking and this disease [31].

\section{Pregnancy}

Cigarette smoking results, in a dose-dependent manner, in several complications during pregnancy (e.g. abortion) and lower birthweight babies [32]. One study found that although quitting smoking during pregnancy produced the greatest increase in birthweight, a $10 \%$ reduction in the 
plasma cotinine level was associated with a $17 \mathrm{~g}$ increase in birthweight [33]. Furthermore, babies born to mothers who reduced smoking during pregnancy had consistently higher birthweights than those babies whose mothers continued smoking the same number of cigarettes during pregnancy. In addition, mothers who reduced the number of cigarettes smoked daily during pregnancy did not have a greater risk of low birthweight babies (defined as $<2.5$ $\mathrm{kg}$ at delivery) than nonsmoking mothers [34].

\section{Implications}

It can be concluded from the above data that quitting is the healthiest decision that smokers can make. Nonetheless, as most studies have shown a dose-dependent risk relationship (higher cigarette consumption represents a greater health risk), a significant reduction in cigarette consumption is likely to reduce harm in smokers.

Although there are few direct demonstrations that reduced smoking will lead to improved health in individual patients, the known reversibility of most smoking-induced diseases, the linear dose-dependent risk curves and the absence of any indication of threshold effects, make it likely that such a reduction in smoking may produce health benefits.

\section{Definition of reduced smoking}

Reduced smoking means smoking fewer cigarettes on an individual level. Reduced smoking is not an end goal, but an indicator or an intermediate stage. As the real objective is to decrease smoking-related morbidity and mortality, there is a need to establish a link between a reduction in the number of cigarettes smoked and reduced harm. However, as any direct assessment of the effect of reduced smoking on future mortality and morbidity would be a difficult and long-term undertaking, it may be possible to evaluate the impact of reduced smoking on surrogate or intermediate parameters linked with tobaccorelated diseases.

One method to reduce harm would be to aim for a reduction in the exposure to toxic substances in inhaled smoke. The best, and most specific, assessable compound that could serve as a marker for total smoke intake is $\mathrm{CO}$, either in exhaled air or bound to haemoglobin ( $\mathrm{COHb}$ ). Thiocyanate, which can be sampled from saliva, also correlates reasonably well with the amount of tobacco smoked. In contrast, the number of cigarettes per day, the easiest measurement, does not correlate completely with the intake of toxic substances; when cigarette smoking is decreased, the remaining cigarettes are often smoked more intensively [35], so the reduction in exhaled CO is usually about $20 \%$ less than the reduction in cigarettes [13, 35]. Thus, a $50 \%$ reduction in daily cigarette consumption would translate into a $30 \%$ reduction in the intake of toxic substances.

\section{Review of reduced smoking studies using nicotine replacement}

Two studies have investigated the utility of nicotine replacement medications to reduce smoking $[13,36]$.
The first study enrolled 15 healthy heavy smokers ( $>40$ cigarettes.day ${ }^{-1}$ ) who were not interested in quitting. Whether these smokers wanted to reduce the number of cigarettes smoked was not stated [13]. All smokers had normal pulmonary function and bronchoscopy status at baseline. The study goal was to reduce the number of cigarettes smoked daily by $50 \%$, and smokers were instructed to use at least 10 pieces of $2 \mathrm{mg}$ nicotine gum per day for 9 weeks. However, the amount of gum each smoker used was not reported.

Cigarette smoking declined rapidly within the first week, from a mean of 51 to 19 cigarettes $\cdot$ day $^{-1}$; this reduction was maintained for the duration of the study, with no evidence of a loss of treatment effect over time. Mean exhaled CO levels decreased from 49 parts per million (ppm) at baseline to $27 \mathrm{ppm}$ at 4 weeks, and remained at this level until week 9; this represented a reduction of $44 \%$. Of 11 smokers who were followed up, four showed some loss of treatment effect from week 4 to week 9 (measured using exhaled $\mathrm{CO}$ ), but this regression was small. Bronchoscopy at week 9 showed a decline in the composite rating of bronchial friability, erythema, oedema and secretions from 8.5 to 6.5 , a reduction of $24 \%$. The number of cells recovered declined by $20 \%$, from $104 \times$ $10^{6}$ to $73 \times 10^{6}$. The level of neutrophils in bronchial samples declined by $38 \%$, from 21 to $13 \%$, and a similar but smaller decrease was observed in alveolar samples. A significant reduction in neutrophil elastase was also reported [13].

This study is important because it shows that, with minimal input from the investigators, heavy smokers were able to significantly reduce the daily number of cigarettes, and that this reduction was maintained for 2 months. Another important finding was that, in addition to reducing the intake of toxic substances, physiological indicators of lower respiratory function were improved, even in healthy subjects.

However, as the study did not include a placebo control, it cannot be concluded that nicotine was responsible for the reduced intake of toxic substances. In addition, as the study involved a selected population of heavy smokers, it is difficult to generalize the results.

In the second study, 143 Swedish smokers unable to/ uninterested in quitting but who were willing to reduce smoking, were allowed to familiarize themselves with the nicotine gum, patch, tablet, spray and inhaler for 1 week [36]. The smokers were then divided into two groups; in the first group, smokers could choose any one of the nicotine preparations to aid smoking reduction over a period of 2 weeks. In the second group, smokers were randomly assigned to a nicotine preparation chosen by the investigator. After 2 weeks, the groups crossed over. Smokers were not given a target daily number of cigarettes but were asked to reduce smoking as much as possible, while smoking enough "to feel comfortable". At study entry, the mean consumption was 23 cigarettes.day ${ }^{-1}$, and the mean score on the Fagerström Test for Nicotine Dependence was 7.0, indicating that these smokers were much more dependent than the average Swedish smoker.

During the first week, when all five medications could be used, smoking decreased by $37 \%$ from 23 to 14 cigarettes.day ${ }^{-1}$, and mean exhaled $\mathrm{CO}$ levels were reduced from 23 to $18 \mathrm{ppm}$ (a reduction of 19\%). During the two 2 week study periods, the overall consumption continued to 
decrease to 10 cigarettes $\cdot$ day $^{-1}(-56 \%)$ and the overall $\mathrm{CO}$ level decreased to $15 \mathrm{ppm}(-35 \%)$. However, the reductions in cigarettes smoked daily and $\mathrm{CO}$ levels were greater when the smokers could choose their own nicotine medication.

Salivary cotinine levels did not change over time and remained near $350 \mathrm{ng} \cdot \mathrm{mL}^{-1}$ throughout the study. Nicotine withdrawal symptoms did not increase as smoking was reduced. During the study, seven smokers (5\%) quit smoking. Surprisingly, $93 \%$ of smokers reported that their desire to stop smoking had increased as a consequence of participating in the study, and $92 \%$ considered reduced smoking to be a good method for ultimately stopping smoking [36].

This study confirmed the results obtained in the earlier, smaller study [13], i.e. large reductions in cigarette smoking and exhaled $\mathrm{CO}$ levels can be achieved and maintained despite some compensatory smoking of the remaining cigarettes. In addition: reduction is possible for those who smoke less than one packet daily; nicotine exposure did not increase despite concomitant use of cigarettes and nicotine medications; smokers can significantly reduce the number of cigarettes smoked daily without experiencing withdrawal symptoms; and, importantly, motivation to quit was increased with reduced smoking.

\section{Risks of concomitant nicotine medications and reduced smoking}

Use of nicotine medication for reduced smoking may involve long-term use, but there are few existing data on whether the long-term use of these preparations poses any health risks. Nicotine is not considered to be carcinogenic and does not appear to contribute to COPD.

\section{Cardiovascular effects}

With respect to cardiovascular effects, animal studies have shown that high doses of nicotine represent an increased risk factor for coronary disease [37]. However, whether nicotine increases the risk of cardiovascular disease in humans is debatable. For example, it appears that Swedish users of smokeless tobacco (snuff), who ingest similar amounts of nicotine to smokers, do not have a higher incidence of coronary disease than nontobacco users [6]. Moreover, smokers of high-nicotine cigarettes do not experience more coronary artery diseases than those who smoke low-nicotine cigarettes [38]. The adverse cardiovascular effects of smoking have been attributed mainly to $\mathrm{CO}$, oxidant gases and glycoproteins, rather than nicotine $[29,33]$.

Only one study, the Lung Health Study [39], has investigated the long-term use of nicotine medications. One of the aims of this study, which involved 3,000 smokers, was to examine the effect of smoking cessation on pulmonary disease. All smokers were strongly encouraged to stop smoking with the aid of nicotine gum. However, after $1 \mathrm{yr}$, $40 \%$ of those who had quit smoking and $25 \%$ of those still smoking were still using the gum. In addition, $10 \%$ of all participants used nicotine gum for the entire $5 \mathrm{yr}$ study period. There was no increase in the incidence of cardiovascular events either in those who had achieved absti- nence but continued use the gum or, more importantly, those who continued to smoke with concomitant gum use. On the basis of these results, the investigators concluded that long-term use of nicotine gum appears to be safe [39].

Similarly, a short-term (10 week) study involving 584 smokers with active cardiac disease found that transdermal nicotine did not increase the incidence of cardiovascular events compared to placebo [40].

\section{Pregnancy}

The other area of concern is the use of nicotine during pregnancy [41]. It is unclear whether the adverse effects of smoking during pregnancy are caused by vasoconstriction of the placental artery by nicotine or foetal hypoxia as a result of CO delivery to the foetus [42]. Although nicotine supplied as gum does slightly increase some indicators of foetal distress, postmarketing surveillance has shown no increase in perinatal events either with the gum or the patch $[42,43]$. This may be because nicotine obtained from the gum or patch produces much more gradual and lower venous nicotine levels than the rapid, high arterial levels achieved with cigarettes [38].

\section{At whom should reduced smoking be targeted?}

Reduced smoking may be indicated in those individuals: 1) who are failing in cessation attempts. Reduced smoking could be suggested for failed quitters until they are motivated to attempt to quit again; 2) who would like to quit but are unable to do so; and 3) who do not want to quit but want to reduce smoking.

It is particularly important for physicians to motivate highly nicotine-dependent smokers to contemplate cessation or reduced smoking, since these individuals have a higher risk of developing smoking-related diseases, such as cancer [44, 45] and COPD [46]. Another important group to target is smokers already suffering from tobaccorelated disease(s) who are unable to quit smoking.

To what extent low nicotine-dependent smokers will respond to reduced smoking using nicotine replacement remains to be clarified, as obtaining nicotine may not be the primary reason for smoking in these individuals.

\section{Practical implementation of reduced smoking}

With respect to the practical management of reduced smoking, baseline smoking values must by ascertained, preferably using exhaled $\mathrm{CO}$ or plasma $\mathrm{COHb}$ levels. Alternatively, plasma nicotine and/or cotinine (the major metabolite of nicotine, with a much longer half-life) levels could also be used.

Smokers should be fully informed about reduced smoking, and the end goals (complete cessation for those wishing to quit and a significant reduction in the number of cigarettes smoked daily for those not yet ready or motivated to quit) should be discussed. A common goal for both groups could be a $50 \%$ reduction in the number of cigarettes smoked daily within a few weeks of commencement. Smokers need time to adjust to their nicotine replacement medications, and it is important that full 
information is provided on the use of the various preparations available. It is preferable to allow smokers to try different nicotine preparations and select the one they feel to be most helpful on an individual basis. Although none of the existing nicotine replacement medications is more effective than the others, the utility of different preparations may vary across smokers. In addition, long-term compliance is likely to be better if individuals feel comfortable with their chosen preparation.

Although nicotine patches are convenient to use, they may not be the ideal preparation for use in reduced smoking programmes. Firstly, patches may not satisfy those smokers who want a direct and discrete effect following nicotine administration. A continuous infusion of nicotine will not be capable of producing significant subjective effects. Secondly, concomitant use of the nicotine patch and cigarettes may result in more nicotine being ingested because of the development of tolerance to the continuous low plasma levels of nicotine achieved with the patch. From the point of view of safety, it may be better to avoid the concurrent entry into the circulation of nicotine from two devices (patch plus cigarettes). As it is unlikely that smoking will take place at the same time as the other nicotine preparations (gum, nasal spray, oral inhaler and tablet) are being used, these preparations are more suitable for reduced smoking programmes.

Finally, smokers should be encouraged to use their preferred nicotine replacement medication frequently, in order to facilitate smoking fewer cigarettes. If a substantial reduction in the number of cigarettes smoked daily has not been accomplished within a few weeks, the treatment may need to be re-evaluated.

\section{What can be gained from reduced smoking?}

One study that investigated the related concept of exposure reduction found that diminishing the tar yield of cigarettes in the UK had a moderately beneficial effect on mortality from ischaemic heart disease and lung cancer [3].

It has recently been estimated that a $1 \%$ reduction in smoking in Austria could, 10 yrs after implementation, reduce the number of male deaths from lung cancer by 14 per annum. A 50\% reduction in smoking would, therefore, prevent 700 premature male deaths from lung cancer. Inclusion of female smokers and other tobacco-related diseases suggests that, even in a country with a small population, thousands of premature deaths could be prevented if the number of cigarettes smoked per individual could be halved (M. Kunze, personal communication).

A similar estimation based on Swedish epidemiological data found that if the number of cigarettes smoked daily could be reduced by $50 \%$, this would result in a $31 \%$ reduction in male lung cancer mortality after 15 yrs [47]. Extrapolation of these Austrian and Swedish results to the entire European Union suggests that at least 100,000 premature deaths from tobacco-related diseases could be prevented if individual smoking could be reduced by $50 \%$. If this goal seems optimistic, even a reduction of $1 \%$ would save 1,000 lives.

It is very difficult to estimate potential health gains in individuals who reduce smoking. A $50 \%$ reduction of smoking in the community may not correspond to the same risk reduction in an individual smoker who cuts cigarette smoke intake by $50 \%$, i.e. if two smokers with the same toxic intake reduce smoking by $50 \%$ and another smoker quits totally, it is not known whether the gain to society will be the same. Furthermore, how do two reductions of the same absolute magnitude, one from 20 to 15 cigarettes daily and the other from five to zero cigarettes daily, compare in terms of risk reduction? More research is, therefore, needed to answer these and other such questions.

The use of nicotine replacement medications to aid reduced smoking is not likely to reduce these potential health gains, given that nicotine obtained from these preparations does not appear to be implicated in the development of cancer or COPD, and poses only a minimal risk for cardiovascular disease.

Finally, although these calculations are speculative, they may underestimate the potential health gains to be made from reduced smoking.

\section{Summary and conclusion}

Societies have not yet generally been successful in controlling the tobacco epidemic and its sequelae. Existing strategies have had, at best, a moderate impact on tobacco-related harm, e.g. although the incidence of lung cancer has started to level out in many countries, decreased incidences are rare. There is, therefore, a need for new strategies to be explored.

Nicotine replacement appears to present a logical opportunity to reduce tobacco-related harm, given that most smokers smoke in order to obtain nicotine and that no untoward events have been observed in smokers who use concomitant nicotine replacement medications. It is interesting to note that Sweden, which has the lowest incidence of tobacco-related harm among developed countries [48], has unintentionally been used as a reduced smoking paradigm. Sweden has relatively few smokers; in 1996, only $18 \%$ of Swedish adults were daily smokers (L. Ramström, personal communication). However, approximately $28 \%$ of adult Swedes use nicotine daily, the explanation being that approximately one third of all nicotine consumed is in the form of moist snuff ("snus"). A recent study of Swedish physicians, whose tobacco use reflects that of the general population, found that $6 \%$ of physicians smoked, $10 \%$ used snuff, and $9 \%$ used nicotine replacement medications [49]. The practice of using snuff has not been found to significantly increase mortality. Thus, reduced smoking and/or abstinence combined with alternative nicotine delivery devices appears to be an un-tapped resource in the quest to reduce tobacco-related harm.

Interestingly, the use of snuff in Sweden allowed heavily dependent smokers to quit [50]. It is known that in smoking cessation programmes, nicotine replacement therapy is of most benefit to highly dependent smokers. If this is also true for reduced smoking, the potential health benefits may be even greater than those estimated because in these analyses all smokers were considered equally dependent ([47], and M. Kunze, personal communication). However, the incidence of tobacco-related lung cancer is higher among highly dependent smokers [44]. A higher degree of nicotine dependence also appears predictive for development of COPD [46], and head and neck cancer 
[45]. Although it remains to be proved, it seems possible that smokers with the highest risk of developing tobaccorelated disease could benefit most from using nicotine replacement medications as an aid to reduce smoking.

At a community level, various actions can encourage reduced smoking including higher taxes on cigarettes and prohibiting smoking, at work and in public places. On an individual level, reduced smoking spontaneously occurs, e.g. after a failed cessation attempt; however, this occurs infrequently and is seldom maintained. It may be feasible for low nicotine-dependent smokers who are strongly motivated to reduce smoking to do so without nicotine replacement, but it is unlikely that highly dependent and less motivated smokers will be able to succeed.

There is also a need for more effective nicotine replacement medications. In order to reach smokers who are indifferent to changing their smoking habits, the alternative nicotine delivery preparations must be at least as effective as cigarettes. In this context, Swedish moist snuff is a good example of an effective product that has contributed to a reduction in smoking-related harm [50].

In conclusion, nicotine obtained from nicotine replacement medications is safer than that obtained from cigarettes, and offers highly dependent smokers an opportunity to control their smoking. Reduced smoking is possible and may be enhanced with nicotine replacement medications. Certain groups of smokers may be better candidates for reduced smoking programmes. As tobacco-related harm depends on the number of cigarettes consumed, reduced smoking may result in huge gains in public health. Finally, reduced smoking may represent the first step towards cessation for those smokers who are not ready or willing to quit.

\section{References}

1. World Health Organization. Tobacco Alert 199; World No-Tobacco Day. Special Issue.

2. Reid DJ, McNeill AD, Glynn TJ. Reducing the prevalence of smoking in youth in Western countries: an international review. Tobacco Control 1995; 4: 266-277.

3. Tang J, Morris J, Wald N, Hole D, Shipley M, Tunstall-Pedoe $\mathrm{H}$. Mortality in relation to tar yield of cigarettes: a prospective study of four cohorts. BMJ 1995; 311: $1530-1533$.

4. Pauly JL, Streck RJ, Cummings MK. US patents shed light on Eclipse and future cigarettes. Tobacco Control 1995; 4: 261-265.

5. Lewin F. Epidemiological aspects of oral cancer: a Swedish perspective (Abstract). International Society for Oral Oncology, 11th Annual Meeting, Stockholm, June 13-15, 1996.

6. Huhtasaari F, Asplund K, Lundberg V, Stegmayr B, Wester P. Tobacco and myocardial infarction: is snuff less dangerous than cigarettes? BMJ 1992; 305: 1252-1256.

7. Bolinder G, Alfredsson L, Englund A, de Faire U. Smoke-less tobacco use and increased cardiovascular mortality among Swedish construction workers. Am J Public Health 1994; 84: 399-404.

8. O'Brien V. Nicorette usage and attitude study, Europe 1996. The Decision Shop, London, July 1996.

9. Giovino GA, Shelton DM, Schooley MW. Trends in cigarette smoking cessation in the United States. Tobacco Control 1993; 2 (Suppl.): 3-16.

10. World Health Organization. Guidelines for controlling and monitoring the tobacco epidemic. Tobacco or Health
Programme 1996, Geneva.

11. US Department of Health and Human Services. The health consequences of smoking: nicotine addiction. A report of the Surgeon General, Public Health Service, Rockville, Maryland, 1988.

12. Russell MAH. The future of nicotine replacement. $B J$ Addiction 1991; 86: 653-658

13. Rennard S, Daughton D, Fujita J, et al. Short-term smoking reduction is associated with reduction in measures of lower respiratory tract inflammation in heavy smokers. Eur Respir J 1990; 3: 752-759.

14. Hoffman D, Wynder EL, Hecht SS. Chemical carcinogens in tobacco. In: Bannasen A, ed. Cancer Risks: Strategies for Elimination. New York, Springer-Verlag, 1988; pp. 101-113.

15. US Department of Health and Human Services. The health consequences of smoking: cancer. A Report of the Surgeon General, Office on Smoking and Health. DHHS Publication No. 82-50179, 1982.

16. US Department of Health, Education and Welfare. The health benefits of smoking cessation. A report of the Surgeon General, Office on Smoking and Health. DHHS publication No. 8:416; 1990.

17. Garfinkel L, Stellman SD. Smoking and lung cancer in women: findings in a prospective study. Cancer Res 1988; 48: 6951-6955.

18. US Department of Health and Human Services. Smoking and Health. A report of the Surgeon General. Office on Smoking and Health. PHS Publication. No. 79-50066; 1979.

19. Hirayama T. A large scale cohort study of the relationship between diet and selected cancer of digestive organs. In: Bruce WR, Correa P, Lipkin M, et al., eds. Gastrointestinal Cancers: Endogenous Factors. Banbury report. New York, Cold Spring Harbour Laboratory, 1981; 7: p. 409.

20. Licciardone JC, Brownson RC, Chang JC, Wilkins JR. Uterine cervical cancer risk in cigarette smokers: a metaanalytic study. Am J Prev Med 1990; 6: 274-291.

21. Daling JR, Weiss NS, Hislop TG, et al. Sexual practices, sexually-transmitted diseases and the incidence of anal cancer. N Engl J Med 1987; 317: 973-977.

22. Brinton LA, Masca PC, Mallin K, et al. Case-control study of cancer of the vulva. Obstet Gynecol 1990; 75: 859-866.

23. Hellberg D, Valentin J, Eklund T, Staffan N. Penile cancer: is there an epidemiological role for smoking and sexual behaviour? Br Med J 1987; 295: 1306-1308.

24. Szarewski A, Jarvis MJ, Sasieni P, et al. Effect of smoking cessation on cervical lesion size. Lancet 1996; 347 : 941-943.

25. Pulera N, Petruzzelli S, Celi A, et al. Presence and persistence of serum anti-benzo(a)pyrene diolepoxide-DNA adduct antibodies in smokers: effects of smoking reduction and cessation. Int J Cancer 1997; 68: 1-5.

26. US Department of Health and Human Services. The health consequences of smoking: chronic obstructive lung diseases. A report of the Surgeon General. Rockville, Maryland, 1984.

27. Lebowitz M, Burrows B. Quantitative relationship between cigarette smoking and chronic productive cough. Int J Epidemiol 1977; 6: 107-113.

28. Fletcher C, Peto R, Tinker C. The natural history of chronic bronchitis and emphysema: an eight year study of early chronic obstructive disease in working men in London. New York, Oxford University Press, 1976.

29. Dockery D, Speizer F, Fernis B, Ware J, Louis T, Spiro A. Cumulative and reversible effects of lifetime smoking on 
simple tests of lung function in adults. Am Rev Respir Dis 1988; 137: 286-292.

30. US Department of Health, Education and Welfare. The consequences of smoking. A report of the Surgeon General. Cardiovascular Disease. Office on smoking and health. DHHS publication No. PHS 84- 50.204, 1984.

31. Klag M, Welton P. Risk of stroke in male cigarette smokers. N Engl J Med 1987; 316: 628-629.

32. Wald N, Hackshaw AK. Cigarette smoking: an epidemiological overview. In: Doll R, Drofton J, eds. Tobacco Health. Br Med Bull 1996; 52: 3-11.

33. Haddow JE, Knight GJ, Kloza EM, et al. Cotinineassisted intervention in pregnancy to reduce smoking and low birthweight delivery. Br J Obstet Gynaecol 1991; 98: 859-865.

34. Li CQ, Windsor RA, Perkins L, et al. The impact on infant birthweight and gestation age of cotinine-validated smoking reduction during pregnancy. J Am Med Assoc 1993; 269: 1519-1524.

35. Foulds J, Stapleton J, Feyerabend D, et al. Effect of transdermal nicotine patches on cigarette smoking: a double-blind cross-over study. Psychopharmacology 1992; 106: 421-427.

36. Fagerström KO, Tejding R, Westin A, Lunell E. Aiding reduction of smoking with nicotine replacement medications: a strategy for the hopeless? Eur Respir J 1996; 9 (Suppl. 23): 170s.

37. Benowitz NL. Nicotine and coronary disease. Trends Cardiovasc Med 1991; 1: 315-321.

38. Hughes JR. Risk/benefit of nicotine replacement in smoking cessation. Drug Safety 1993; 8: 49-56.

39. Murray RP, Bailey W, Daniels K, et al. Safety of nicotine polacrilex gum used by 3,094 participants in the Lung Health Study. Chest 1996; 109: 438-445.

40. Joseph AM, Norman SM, Ferry LH, et al. The safety of transdermal nicotine as an aid to smoking cessation in patients with cardiac disease. $N$ Engl J Med 1996; 335: 1792-1798.

41. Benowitz NL. Nicotine replacement during pregnancy. JAMA 1991; 266: 3174-3177.

42. Hoechst Marion Roussell. OTC Nicorette Data Summary. Presented at the Non-Prescription Drugs and Drug Abuse Advisory Committees of the Food and Drug Administration, 1995.

43. OTC Nicoderm Data Summary. Presented at the Non-Prescription Drugs Advisory Committee of the Food and Drug Administration, 1996.

44. Kunze U, Schoberberger R, Fagerström KO, et al. High nicotine dependence among lung cancer patients $(\mathrm{Ab}$ stract). Eur Respir J 1996; 9 (Suppl. 23): 176s.

45. Gritz ER, Carr CR, Rapkin D, et al. Predictors of longterm smoking cessation in head and neck cancer patients. Cancer Epidemiol Biomarkers Prev 1993; 2: 261-270.

46. Eugen-Obradovic I, Miric-Milla M, Bosnjak-Petrovic V, et al. The frequency of smokers and nicotine addiction grade of active smokers with chronic obstructive pulmonary disease. Tuber Lung Dis 1995; 76: 103-106.

47. Wiklund K, Hakulinen T, Sparén P. Prediction of cancer mortality in the Nordic countries in 2005: effects of various interventions. Eur J Cancer Prev 1992; 1: 247-258.

48. Peto R, Lopez AD, Boreham J, et al. Mortality from smoking in developed countries, 1950-2000. Oxford, Oxford University Press, 1994; p. 668.

49. Bolinder G, Himmelmann L. Färre röker men fler snusar. Läkartidningen 1996; 93: 4437-4444.

50. Ramström L. Reducing tobacco-related diseases: alternative approaches. Workshop organised by the International Council on Alcohol and Addictions Section on Tobacco Dependence, Amsterdam, July 1996. 